\title{
SPAANSE KINDEREN. LOS NIÑOS ESPAÑOLES EXILIADOS EN BÉLGICA DURANTE LA GUERRA CIVIL. EXPERIENCIA PEDAGÓGICA E HISTORIAS DE VIDA
}

\author{
Spaanse Kinderen. Children Spanish Exiles in Belgium during the Civil \\ War. Learning Experience and Life Stories
}

Andrés Payà Rico

Universitat de València

andres.paya@uv.es

Fecha de recepción: 18-II-2013

Fecha de aceptación: 26-II-2013

Resumen: El presente artículo narra la experiencia pedagógica llevada a cabo el curso 2011-2012 por el autor en la Universiteit Gent (Bélgica), en la cual se intentó recuperar la memoria histórico-pedagógica mediante la historia oral. La búsqueda y análisis documental en archivos belgas de correspondencia, fotografías y dibujos, así como el empleo de entrevistas e historias de vida a los «nińos» exiliados en Gante durante la Guerra Civil española, son las fuentes empleadas para el estudio de estas historias de la infancia. Una investigación histórica compartida con los estudiantes de Pedagogische Wetenschappen (Ciencias de la Educación) que se convirtió en una verdadera experiencia de aprendizaje histórico-educativo.

Palabras clave: exilio, historia oral, historia de la infancia, historia social, historia de la educación, Bélgica.

Авstract: This paper explains the pedagogical experience conducted during 2011-2012 by the author at the Ghent University (Belgium), in which he tried to recover the historical memory through oral history teaching. The search and document analysis in Belgian correspondence files, photographs and drawings, and the use of interviews and life stories of "children" exiled in Ghent during the Spanish Civil War, are the sources used for the study of these childhood stories. This is a historical research 
shared with students of Pedagogische Wetenschappen (Educational Sciences) that became a real learning experience of historical and educational learning.

Keywords: exile, oral history, history of childhood, social history, history of education, Belgium.

\section{LOS NIÑOS EXILIADOS EN BÉLGICA DURANTE LA GUERRA CIVIL ESPAÑOLA (1937-1939)}

El inicio y el posterior recrudecimiento de la Guerra Civil española provocaron un importante flujo migratorio y desplazamientos internos de la población. Las niñas y los niños fueron trasladados a zonas más seguras y alejadas del peligro de los bombardeos, fundamentalmente a Catalunya y Valencia ${ }^{1}$. En las colonias escolares, los hogares de acogida o en casas de familiares, la infancia española pudo huir durante un tiempo del horror, el hambre y la barbarie bélica. Paralelamente, otros optaron por marchar al extranjero, una salida que fue creciendo conforme avanzaba la guerra y se convirtió en masiva con el fin de la misma. De los puertos de Bilbao, Santander, Gijón, Valencia y Alicante se fletaron numerosos barcos que trasladaban a estos niños hacia un lugar más seguro, mientras que la vía terrestre era la utilizada para aquellos que lo hacían desde Cataluña. Diferentes países fueron el escenario de este exilio infantil, acogiendo a un gran número de niños y niñas en colonias y domicilios de particulares que se movilizaron a tal efecto. De este modo, Inglaterra, Suiza, Dinamarca, la Unión Soviética, México, Francia y Bélgica ${ }^{2}$ se convirtieron en los nuevos hogares de estos niños españoles. Se ha investigado y publicado mucho al respecto $y$, afortunadamente en las últimas décadas, fruto del interés historiográfico por la historia social, la historia de la infancia y la historia oral ${ }^{3}$, se han recuperado muchas historias de vida de estos niños y niñas que tuvieron que marchar de su país. Nos gustaría hacer aquí nuestra particular aportación, fijando la atención en aquellos niños españoles que se exiliaron en Bélgica ${ }^{4}$, especialmente en aquellos que fijaron su residencia en la ciudad de Gante.

1 ESCRIVÀ MOSCARDÓ, Cristina y MAESTRE MARIN, Rafael (2011) De las negras bombas a las doradas naranjas. Colonias escolares 1936-1939, Valencia: L'Eixam Edicions.

2 BERGUER, Geraldine (1991) Les enfants de la Guerre Civile espagnole. Chronique du périple des enfants espagnols réfugiés en Bélgique, Mons : École d'Interprètes Internationaux, Université de Mons.

3 BARRENETXEA MARAÑÓN, Igor (2012) «Los niños de la guerra: entre la historia oral, el cine y la memoria» en Historia contemporánea, 45, pp. 741-768.

4 ELOY, Sarah (2005) De Spaanse migratie naar België gedurende de 19de en 20ste eeuw. "Los Niños de la Guerra»: Een casus, Gent: Universiteit Gent. 
Según distintos investigadores ${ }^{5}$, se calcula que alrededor de 5.000 niños y niñas fueron evacuados a Bélgica, previo paso por Francia, durante la Guerra Civil espańola. De estos, un importante contingente (3.300) procedía del País Vasco ${ }^{6}$ y el resto de otras zonas del país (Madrid, Catalunya, Cantabria, Aragón y Asturias, principalmente). Desde 1937, con la ayuda como escolta de la armada inglesa y el beneplácito del gobierno francés, la mayoría de las evacuaciones se produjeron en grandes barcos, entre los que destacan el "Habana», "Goizeko-Izarra» y "Cabo Corona», con destino a los puertos de La Pallice, Pauillac o Bayonne. Asimismo, de Catalunya también partieron hacia Francia vía terrestre (camiones y ferrocarril) o marítima un importante número de niños en el llamado "éxodo final» entre enero y febrero de 1939. Una vez en territorio francés a los niños y niñas españoles se les realizaba un examen médico y se les proporcionaban vacunas, alimento y ropas adecuadas. Unos pasaron un tiempo en colonias francesas ${ }^{7}$ antes de ser trasladados en tren a Bélgica, destacando por sus instalaciones y el número de nińos acogidos la «Maison Heureuse» en la Ille d'Olèron, junto a «Domaine de Magenta» en Moliets-et-Maa, «Le Hameau des Écureuils» en Capbreton, «Val d’Or» en Saint Cloud o las establecidas en Guéthary o Arraute-Charitte. Otros fueron trasladados directamente a las colonias de la costa belga, como «Home Emile Vandervelde» en Oostduinkerke, «Home de Vacances» en Heyst-aan-Zee, «L'Age Heureux» en Niewport, «Maison de Repos» en Groenendijk, o bien fueron distribuidos en las mismas estaciones de ferrocarril belgas a las familias de acogida.

Puesto que el Estado belga, más allá de alguna colaboración puntual o de pequeñas subvenciones económicas, permaneció al margen, tuvo que ser la ciudadanía, por medio de las familias, los sindicatos y las asociaciones nacionales e internacionales quien protagonizara esta acogida. Uno de los colectivos belgas que pusieron todo su empeño en esta tarea, fue el partido socialista Parti Ouvrier Belge-Belgische Werkliedenpartij (POB-BWP) ${ }^{8}$ quien a través de las Femmes Prévoyantes Socialistes junto a otros partidos y sindicatos de izquierdas organizaron el Comité National pour l'Hébergement des Enfants Espagnols en Belgique (CNHEEB), con sede en Bruselas y dirigido por August de Block (secretario del POB-BWP) junto a Isabelle Blume y Edward Vlaeminck. Así, a partir de abril de 1937 comenzó la labor del CNEEEB gracias al cual se estima que fueron acogidos unos 2.500

5 LABAJOS-PÉREZ, Emilia y VITORIA-GARCÍA, Fernando (1997) Los niños españoles refugiados en Bélgica (1936-1939), Namur: Asociación de los niños de la guerra, p. 45; ALONSO CARBALLES, Jesús J. (1998) 1937. Los niños vascos evacuados a Francia y Bélgica. Historia y memoria de un éxodo infantil, 1936-1940, Bilbao: Asociación de nińos evacuados el 37, p. 152.

6 Cfr. BELL, Adrián (2011) Sólo serán tres meses: los niños vascos refugiados en el exilio, Barcelona: Plataforma; y LEGARETTA, Dorothy (1987) The Gernica Generation. Basque refugee children of the Spanish Civil War, Reno: University of Nevada.

7 ALONSO CARBALLÉS, Jesús J. (1998) 1937. Los niños..., Op. Cit., pp. 158-159.

8 EECKHOUT, Tania (1987) «De hulp aan republikeins Spanje uitgaande van de BWP, afdeling Gent-Eeklo, meer specifiek de opvang der Spaanse kinderen» en Revue Belge d'Histoire Contemporaine-Belgisch Tijdshrift voor Nieuwste Geschiedenis, Vol. XVIII, no 1-2, Bruxelles, pp. 240-246 

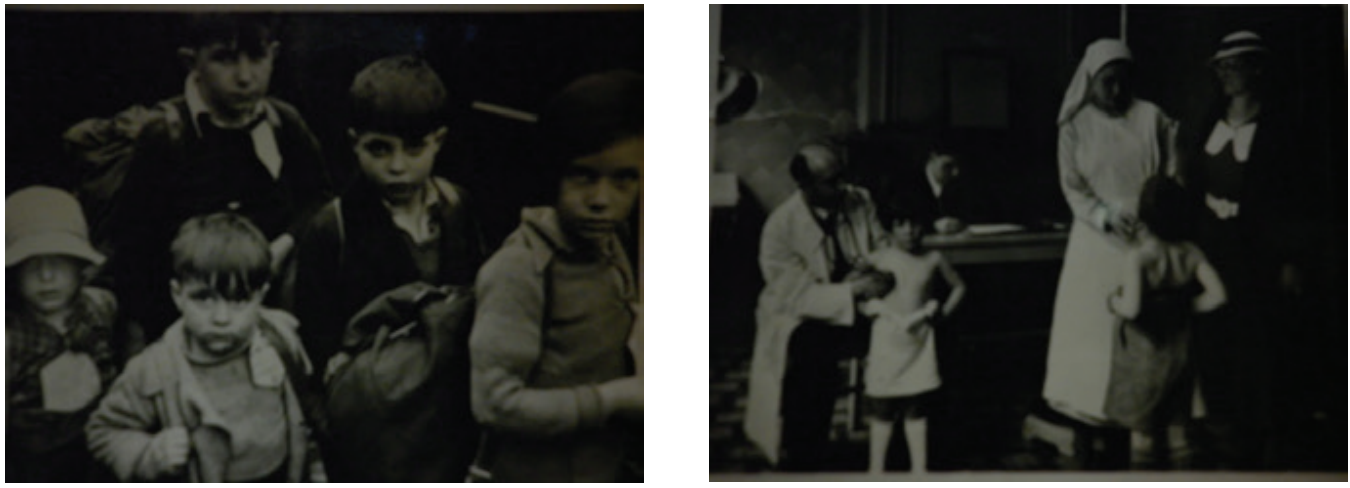

(Izquierda) Niños españoles subiendo al tren, Archivo Amsab. (Derecha) Examen médico en la colonia, Archivo Amsab

niños en colonias y familias belgas. Tras una breve estancia en Francia, eran trasladados en tren a colonias de la costa (Emile Vandervelde, Lys Rouge o L'Age Heureux) donde durante dos o tres semanas intentaban mejorar su deplorable estado de salud antes de ser adoptados o apadrinados temporalmente por alguna familia trabajadora.

De manera paralela a la movilización de los partidos obreros, los sectores católicos belgas también emprendieron una importante iniciativa de acogida, en este caso, de niños y niñas procedentes del País Vasco?. El 27 de abril de 1937 el Obispo de Vitoria Mateo Múgica, abrumado por los acontecimientos bélicos en Euskadi, decide escribir una carta al Cardenal Arzobispo de Malinas Van Roey demandando su ayuda para acoger a nińos católicos vascos, como reacción a la iniciativa de los movimientos obreros ${ }^{10}$. La respuesta de Van Roey fue rápida y su secretario el canónigo Leclef, el vicario general monseñor Jansen junto a José María de Izaurrieta, miembro del Comité de Evacuación del Gobierno de Euskadi en París, se pusieron a trabajar para la creación del Baskisch Kinderwerk-L'Oeuvre des enfants basques. La colaboración de organizaciones católicas como la Jeunesse Ouvrière Chrétienne (JOC), Soeurs de la Charité, Filles de la Charité, Cáritas, la Sociedad Saint Vincent de Paul y, sobretodo, de las familias católicas belgas permitió que un total de unos 1.300 niños y niñas, acompañados de unos treinta sacerdotes y veinte

9 LEGARETTA, Dorothy (1987) «Hospitality to the Basque refugee children in Belgium» en Belgisch tijdschrift voor Nieuwste Geschiedenis, XVIII, pp. 275-288.

10 "Mes enfants! Je suis informé par la presse et par des lettres particulières que de la partie de mon diocèse, qui se trouve encore en plein milieu de la terrible guerre-c'est-à-dire de Bilbao-sont partis et partent pour l'étranger des centaines d'enfants, afin de ne pas tomber victimes de la famine et de la mitraille (...) Je voudrais qu'au moins les enfants qui sont obligés de passer dans votre pays, et qui sont fils de parents très chrétiens dont la plus grande gloire est d'être membres de l'Eglise puissent être reçus dans des centres ou des familles ou leur foi n'ait à courir aucun risque» Archivo del Arzobispado de Malinas, Caja Número 1. 
andereños o maestras vascas, se beneficiaran de esta acogida. La gran mayoría de estos niños residieron en hogares de familias católicas belgas, si bien por diferentes motivos (nińos no adaptados a las familias, grupos de hermanos que no habían podido ser alojados en la misma familia o niños de mayor edad) permanecieron en colonias católicas creadas en Schoten, Duffel o Kortrijk ${ }^{11}$.

Estas dos iniciativas encabezadas por los socialistas y los católicos belgas respectivamente, acogieron un total 3.800 nińos y niñas, el resto, unos 1.200, se beneficiaron de diferentes acciones humanitarias de organizaciones de distinta índole que permitieron huir de la Guerra a una parte de la infancia española. Entre estas iniciativas menos numerosas pero igualmente importantes se encuentra la emprendida por el Departamento de Asistencia Social del Gobierno de Euskadi y el Comité Belga-Vasco ${ }^{12}$ de la embajada de España en Bruselas, quienes pusieron en marcha el Home Belgo-Basque en Marchinlez-Huy (Liège), donde los niños convivían con mujeres y ancianos refugiados. Muy significativa fue también la labor de solidaridad emprendida por la Cruz Roja de Bélgica por medio del Comité Belge d'Asistance aux Enfants d'Espagne13, creado a tal efecto en el verano de 1937 y que impulsó la creación de hogares de acogida temporal en Duden (Bruxelles) y Sète (Hérault), donde los nińos eran atendidos y se preparaban para la acogida por familias belgas. De otro lado, la solidaridad comunista también se movilizó mediante el Socorro Rojo Internacional, quien además de colaborar con el CNHEEB anteriormente aludido, organizó tres evacuaciones ${ }^{14}$ de niños provenientes de Bilbao y Santander. La sección belga de la Office Internationale pour l'Enfance (OIE) dirigida por Germaine Hannevart también se encargó de la acogida, identificación y distribución de niños refugiados de Asturias, Madrid, Cantabria, Aragón y Cataluña. Finalmente, el Grupo Español para la Defensa de la República, creado por la Casa de España de Bélgica junto al Gobierno de la República española, colaboró también con la acogida en una colonia en Rixensart y la distribución a diferentes familias belgas

Al finalizar la Guerra Civil, la mayoría de los niños fueron repatriados a España, volviendo a una realidad social, política y educativa de posguerra y dictadura muy diferente

11 ALONSO CARBALLÉS, Jesús J. (1998) 1937. Los niños vascos evacuados a Francia y Bélgica. Historia y memoria de un éxodo infantil, 1936-1940, Bilbao: Asociación de nińos evacuados el 37, p. 287.

12 Comité formado por Francisco García Lorca (hermano del poeta Federico García Lorca) en representación de la Embajada de España, Bertelson del Ministerio de Asuntos Exteriores, Núñez de Prado, delegado del Gobierno Vasco y la diputada socialista Isabel Blume.

13 Diario Le Peuple, 9-VII-1937 «L'Oeuvre de la Croix-Rouge de Belgique en faveur des enfants espagnols» y revista Croix Rouge, agosto de 1937 "D’Espagne en Belgique. Les enfants basques à Bruxelles».

14 «Un nouveau contingent de cent cinquante-quatre enfants basques est arrivé hier après-midi à Bruxelles. A nouveau, nos attend la même vision : visages fatigués, et émaciés de gosses qui sortent de l'enfer de Santander et dont les grands yeux bruns et noir reflètent encore l'épouvante des bombardements», diario La Voix du Peuple, 30-VI-1937. 
a la que habían vivido en Bélgica. El retorno fue diferente según los casos, pues si bien las organizaciones católicas facilitaron el regreso de los niños acogidos en las distintas familias, las organizaciones obreras no reconocieron al gobierno franquista y no colaboraron con las repatriaciones, y las instituciones como Cruz Roja o la OIE no pusieron impedimento alguno y ejercieron, en ocasiones, de árbitro o juez neutral. Sea como fuere, la dictadura se encargó de realizar una dura campańa de propaganda a favor de la repatriación de los niños en el exilio ${ }^{15}$, tergiversando la realidad y acusando en la prensa a los organismos e instituciones de acogida de "crimen de la evacuación infantil», «los niños que arrancaron los rojos de su patria», «arrancados por la fuera de los brazos de sus madres», etc. Por diferentes motivos (muerte del padre o la madre, prisión de los padres, la situación económica y política española, las represalias franquistas, petición de los propios padres, o deseo de los padres adoptivos) unos 1.300 nunca volvieron a España o bien regresaron para establecer su residencia en Bélgica de manera permanente. Los testimonios de parte de este grupo de «nińos» $\mathrm{y}$ «nińas» hoy octogenarios o sus familiares que todavía viven en Gante, han sido el objeto del estudio histórico-pedagógico que presentamos a continuación.

\section{RECUPERANDO HISTORIAS DE VIDA. UNA EXPERIENCIA PEDAGÓGICA EN LA UNIVERSITEIT GENT}

Gracias a una ayuda obtenida en la convocatoria de estancias en otras universidades y centros de investigación del Vicerectorat d'Investigació i Politica Cientifica de la Universitat de València y a la licencia de estudios concedida por el Vicerectorat d'Ordenació Acadèmica i Professorat, tuvimos la oportunidad de realizar una estancia de investigación en el Vakgroep Pedagogiek de la Faculteit Psychologie en Pedagogische Wetenschappen de la Universiteit Gent durante los meses de junio a diciembre del 2011 para investigar las historias de vida de los niños exiliados en Bélgica. A lo largo de seis meses profundizamos en el estudio e investigación de esta parte de la historia de la educación española en el exilio, en un largo, intenso y apasionante proceso de investigación y aprendizaje que intentaremos reproducir aquí de la manera más sintética posible.

Coincidiendo con el final del curso académico 2010-2011 en la Universiteit Gent, nos incorporamos en el mes de junio al departamento Vakgroep Pedagogiek dirigido por el profesor Marc Spoelders quien, junto a un magnifico grupo de profesores e investigadores, brindaron una cálida bienvenida al plan de trabajo. Los tres primeros meses, a la espera del inicio del curso 2011-2012 estuvieron dedicados a la investigación documental en archivos e instituciones belgas como el Centre d'Études et de Documentations Guerre et Sociètes

15 MAYORAL GUIU, Miguel y ALONSO CARBALLÉS, Jesús (1993) «La repatriación de los niños del exilio: un intento de afirmación del régimen franquista (1937-1939)» en TUSELL GÓMEZ, Javier (Coord.) El régimen de Franco, 1936-1975: politica y relaciones exteriores, Vol. 1, Madrid: UNED, pp. 341-350. 
Contemporaines de Bruxelles o el Institut d'Histoire Sociale-Institunt voor Sociale Geschiedenis (Amsab-ISG) de Gante, siendo este último una pieza principal en el proceso de investigación. De manera paralela, esta labor archivística fue complementada con la búsqueda de posibles «nińos» $\mathrm{y}$ «nińas» exiliados en Bélgica a quien poder entrevistar con posterioridad, para ello contactamos y visitamos asociaciones y entidades de diversa índole donde encontrar estos protagonistas de las historias de vida: Fondation Joseph Jacquemotte, Consejos de Residentes Españoles (CRE), Federación de Asociaciones de Enseñanza y Centros españoles en Bélgica (FAECEB), Movimiento asociativo de emigrantes españoles en Bélgica, Asociaciones de pensionados y jubilados de Liège y Herstal, Centros asturianos, gallegos, andaluces y Casal Català de Bruxelles, diferentes Hogares españoles... con un resultado bastante dispar.

El análisis de la documentación encontrada y los resultados de los diferentes contactos establecidos, llevaron a replantear la investigación inicial cuyas pretensiones y acotación hubieron de limitarse a los «nińos» residentes en la ciudad de Gante y área metropolitana. A principios de septiembre y con el trabajo bastante adelantado, el profesor de la Universiteit Gent y Commisioner for Children's Rights in Flanders, Bruno Vanobbergen se ofreció a colaborar en el proyecto, mediante la asignatura Kindstudies ${ }^{16}$ del Master in de pedagogische wetenschappen, afstudeerrichting pedagogiek en onderwijskund para el curso 2011-2012. Una asignatura relacionada con la historia de la educación y la cultura, la sociología y la filosofía de la educación, cuyos contenidos versan sobre el «descubrimiento» de la infancia y la historia de la educación social. La pertinencia temática con las líneas de la asignatura permitió que la investigación que finalmente denominamos "Life histories of spanhis children in Ghent» fuese incluida en la programación docente como una de las tres opciones de trabajo para los alumnos de la asignatura. Puesto que los «niños» $\mathrm{y}$ «niñas» o sus familiares belgas ahora hablaban flamenco (neerlandés) y habían olvidado en muchos casos el español, se hacía necesaria la colaboración del alumnado para llevar a cabo las entrevistas y sus transcripciones correspondientes en flamenco.

Junto al profesor Vanobbergen consideramos que esta sería una buena ocasión para la iniciación práctica a la investigación histórico-educativa de los alumnos de la Universiteit Gent, así que en el mes de octubre organizamos un seminario de dos días en Lokeren con el alumnado de Kindstudies para presentarles en diferentes sesiones las tres opciones de trabajos académicos:

1. Kinderen in zee hospitalen (Los niños en los hospitales del mar), dirigido y tutorizado por Bruno Vanobbergen en el cual se proponía la investigación de aquellos niños de principios del siglo XX que eran hospitalizados en sanatorios de la costa belga.

16 <http://www.opleidingen.ugent.be/studiegids/2009/NL/FACULTY/PP/COURSE/ HHPEDG/01000007/INDEX.HTM> Consultado el 2 de febrero de 2013. 
2. Verlaten kinderen, Gentse weeskinderen na WO II (Niños abandonados, huérfanos en Gante tras la Segunda Guerra Mundial), dirigido y tutorizado por Lieselot de Wilde, dedicado a la investigación de la infancia abandonada y marginada.

3. Spaanse kinderen exile (1937-1939), propuesta de trabajo en un power point (cuya traducción al neerlandés de estos y otros documentos de la investigación fue posible gracias a la colaboración del investigador y compañero de la Universiteit Gent, Elias Hemelsoet) junto a la proyección del documental Kinderen van Gernica ${ }^{17}$, de la productora Ambrosia's Tafel, en el que se narra la experiencia de educación para la paz con los alumnos del instituto Sint Vincentius (Gent). Dicho documental fue clave para poder localizar a Manuel Múgica-González, jefe del departamento de formación de la policía local de Gante e hijo de Ángel Múgica-González (niño de la guerra), narrador y promotor del documental en el que, a propósito de la historia de vida de su padre, se lleva a cabo un proyecto docente de educación para la paz con alumnos de bachillerato.

Dicho seminario se complementó al día siguiente con la exposición del trabajo de grupo de una alumna del curso anterior, así como con dos sesiones metodológicas dedicadas a la historia oral y a las fuentes iconográficas, y una última sesión de clarificación de dudas y distribución de los grupos de trabajo. Finalmente, dos motivados grupos de cuatro alumnos y alumnas se animaron a trabajar en las historias de vida de los niños españoles: Lore Arnauts, Cyrina Grimonprez, Elise Ryckebusch y Elle Winten (Grupo 1) y Jelmen Haaze, Ward Vandenberghe, Liesbeth Hoedt y Jorre Van de Sompele (Grupo 2). Para una adecuada contextualización y profundización, se les facilitó la lectura de las dos referencias existentes sobre el exilio de los niños españoles en Bélgica escritas en neerlandés ${ }^{18}$, entretanto quedaban emplazados a un seminario a celebrar en los primeros días del mes de noviembre para presentarles el material recopilado hasta la fecha y la metodología a seguir.

Este segundo seminario tuvo lugar en el Amsab-Instituut voor Sociale Geschiedenis de Gante, un archivo cultural nacional que se encarga de recoger y mantener el registro de los movimientos sociales y figuras progresistas de principios del siglo XIX hasta la actualidad, cuya misión es la de «identificar el patrimonio social progresista, la emanci-

17 Disponible on line en <http://www.ambrosiastafel.be/index.php?id=53> Consultado el 18 de febrero de 2013.

18 CAESTECKER, Frank; ELOY, Sara y PUWELS, Hilde (2007) Los niños: tien vluchtelingenkinder uit de Spaanse burgeroorlog vertellen, Berchem: EPO; y LABAJOS-PEREZ, Emilia; VITORIA-GARCÍA, Fernando y SOUBRY, Ludwine (1994) Los niños: de kinderen van de Spaanse burgeroorlog in België (1936-1939), Gent: Amsab. 


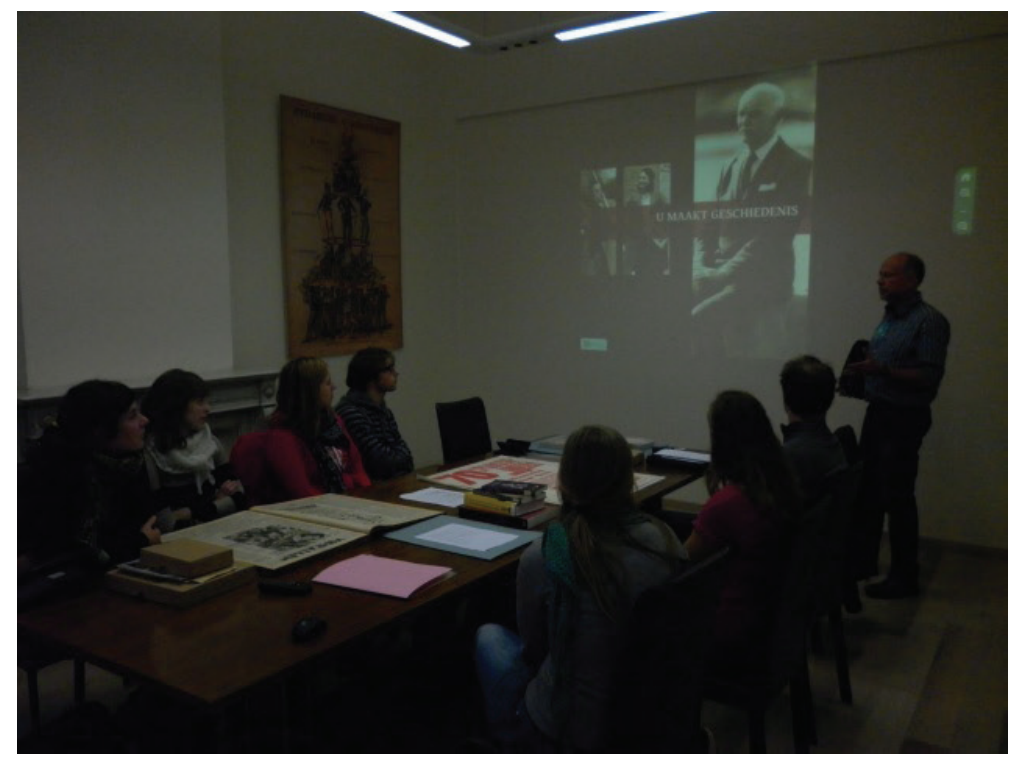

Seminario de trabajo en el Amsab (Gante, 2011)

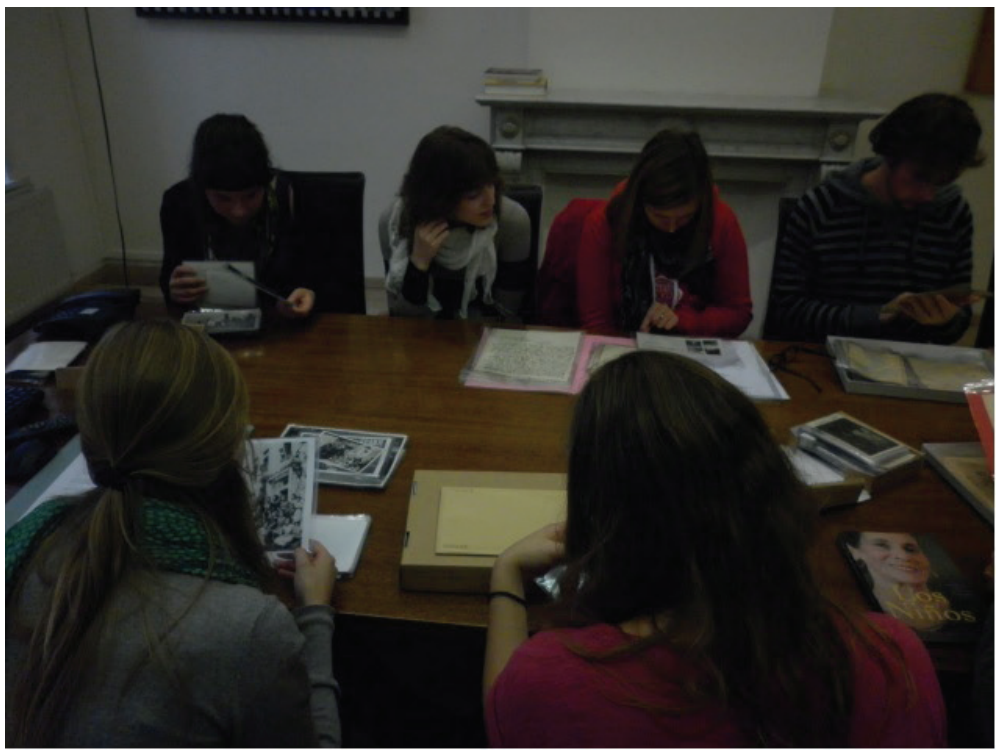

Trabajando con el material, Amsab (Gante, 2011)

pación social y ecológica en Bélgica. Conservar este patrimonio y ponerlo a disposición de cualquier persona interesada. Fomentar y desarrollar la investigación científica en el campo de la historia social. Fortalecer los vínculos con la investigación académica. Poner 
en marcha las iniciativas públicas para elevar activamente el patrimonio» ${ }^{19}$. Gracias a la colaboración del director del archivo Henry Ollivier, la jefa de relaciones públicas Paule Verbruggen y al bibliotecario principal Luc Lievyns, tuvimos acceso a todos los fondos que custodia el Amsab sobre los nińos españoles refugiados en Gante, y pudimos organizar (como muestran las fotografías) un seminario de trabajo con los alumnos sobre los materiales existentes (fotografías, carteles, prensa, correspondencia, filatelia...).

Durante el resto del mes de noviembre los alumnos contactaron telefónicamente con el listado de contactos de los «niños» exiliados en Gante o de sus familias, que habíamos elaborado en los meses anteriores. Un listado difícil de elaborar debido a que las referencias que teníamos de personas entrevistadas en anteriores investigaciones tenían más de 10 años y durante este período de tiempo muchas de ellas habían fallecido. A pesar de estas y otras dificultades, como la poca predisposición de algunos exiliados localizados a ser entrevistados y recordar sus vivencias, finalmente obtuvimos un listado de 20 «niños» o de sus familiares dispuestos a colaborar. Una relación que pudimos elaborar gracias a varias fuentes, como la inserción en la web de noticias locales Gentblogt.be, el contacto con la asociaciones e instituciones anteriormente mencionadas y, sobre todo, gracias a la inestimable colaboración de Manuel Múgica-González, de la policia local de Gante, que nos facilitó varias direcciones y contactos.

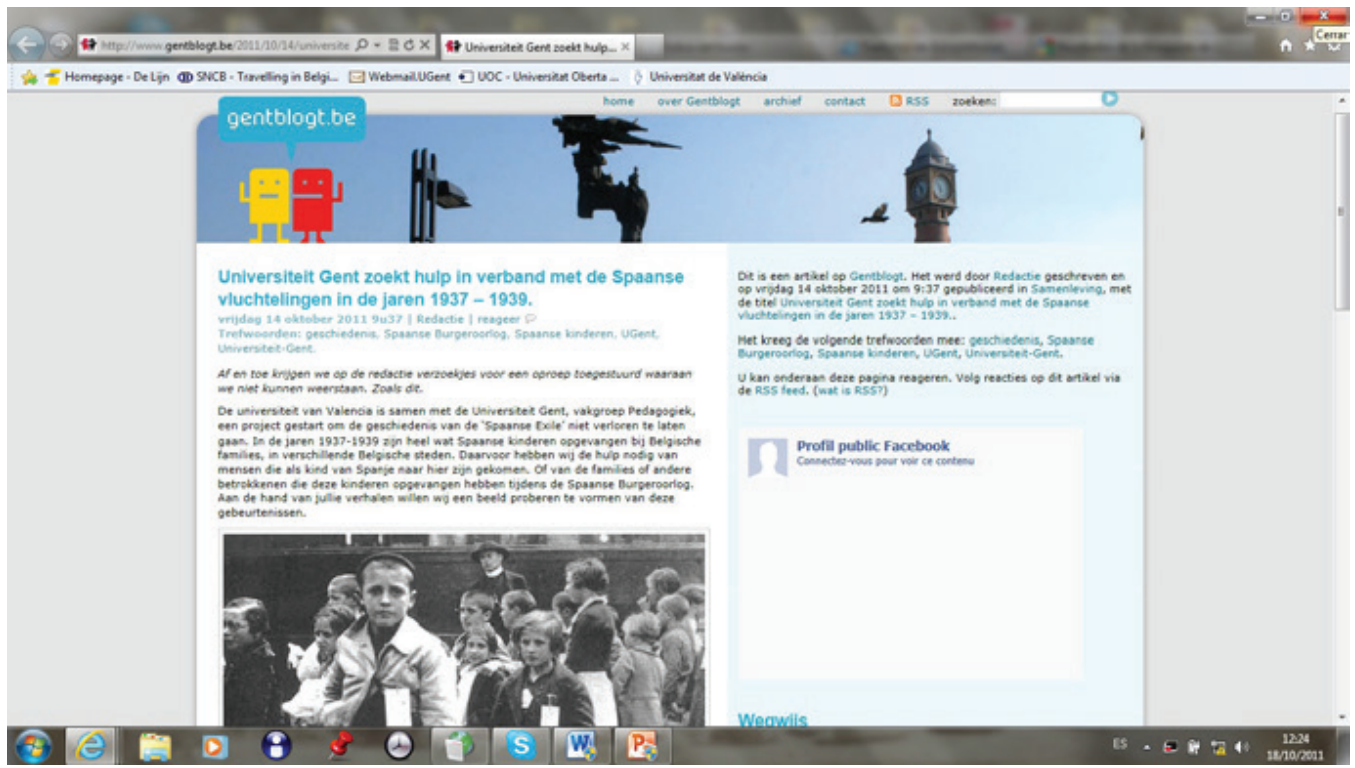

Anuncio publicado en gentblogt.be solicitando el contacto con niños españoles exiliados $(12 / 10 / 2011)$

19 <http://www.amsab.be/> Consultado el 15 de febrero de 2013. 
Así, en las semanas posteriores tuvieron lugar las entrevistas grabadas en audio y transcritas en neerlandés, obteniendo un total de 12 entrevistas que suman más de 18 horas de grabación con las historias de vida de los niños españoles exiliados a Gante. En la primera semana de diciembre tuvimos el último seminario de puesta en común y análisis de resultados, en el que además de la satisfacción por el trabajo realizado y el aprendizaje obtenido, compartimos los aspectos más importantes o destacados, curiosidades, semejanzas y diferencias entre las distintas historias de vida, cuyo contenido relatamos brevemente a continuación.

\section{BALANCE DE LA EXPERIENCIA Y PERSPECTIVAS DE FUTURO.}

La evaluación inicial que se puede hacer tras seis meses de trabajo de investigación documental y de historia oral podemos calificarla como excelente o, al menos, muy grata y satisfactoria. Aunque el análisis de toda la documentación generada requiere un pausado estudio histórico, nos gustaría señalar aquí simplemente el balance obtenido tras el trabajo realizado y las vías o líneas de investigación que se abren a partir de aquí.

En lo concerniente a las historias de vida recopiladas y las horas de grabación transcritas, se hace necesario valorar pormenorizadamente cada uno de los casos y entrevistas, pues si bien existen rasgos comunes a todos ellos, la singularidad de las trayectorias personales y la casuística de cada una de ellas en función de muchos factores (edad, familia de acogida, integración social, educación, etc.) convierten a cada historia de vida en única y singular. El balance final de las entrevistas arroja un resultado de doce historias de vida: cinco «niños» de la guerra (Carlos Pascual Madorran, Elvira Fernández Alonso, Germana Contrera Miravalles, Nestor Folla y Santiago Roldán Aragón), la esposa de un «nińo» (Jacqueline Van Overbeke) y seis hijos de los «niños» exiliados (Patrick Fakkel, Carmen Fakkel, Johny Larrea Betolaza, Manuel Múgica-González, Jenny Folla y Teresa Pérez Diego). Unos entrevistados cuyas narraciones son verdaderamente increíbles y forman parte de la historia viva de nuestro presente más cercano, y a quienes nunca podremos agradecer lo suficiente la colaboración en este ejercicio de memoria histórica. Las entrevistas eran semi-estructuradas y contaban con el siguiente guion o esquema básico distribuido en seis bloques temáticos:

Identificatiegegevens: Naam en voornaam; Plaats en datum van geboorte; Jaren had ik toen ik naar België Datos de identificación: Nombre y apellidos; Lugar y fecha de nacimiento; Años que tenía cuando vino a Bélgica.

De reis: Reizen Spanje-België; Koloniën, accommodatie of voorafgaand aan de verzorgingshuizen te bevorderen; Docenten, volwassenen of broers en zussen die hem vergezelden. El viaje: Viaje España -Bélgica; Colonias, albergues o hogares previos al acogimiento familiar; Profesores, adultos o hermanos que le acompañaron. 
De gastheer: De eerste indruk bij aankomst in België; Organisatie van de gastheer (de Belgische Socialistische Partij, een werk van kardinaal Van Roey...); Grote problemen of problemen te integreren; Algemene vreugde of tevredenheid met de komst. La acogida: Primeras impresiones al llegar a Bélgica; Organización de la acogida (Partido Socialista Belga, Obra del Cardenal Van Roey...); Principales dificultades o problemas de integración; Principales alegrías o satisfacciones con la llegada.

Het gastgezin: Behandeling van het gastgezin; Relatie, doet denken aan de Spaanse familie; Onderwijs en school in België; Leerkrachten en klasgenoten; Sociale integratie, vriendschappen; Games, amusement en recreatie; De tal; De voeding; Gezondheid; Religie. La familia de acogida: Trato de la familia de acogida; Relación, recuerdo de la familia española; La educación y la escuela en Bélgica; Los profesores y los compañeros de clase; Integración social, amistades; Los juegos, el ocio y el tiempo libre; El idioma; La alimentación; La sanidad; La religión.

Het nieuwe leven in België: Bouw een gezin; Aanpassing aan de land eind; Feeling, het verlangen om terug te keren? La nueva vida en Bélgica: Construir una familia; Adaptación final al país; Sentimiento ¿̇eseo de retorno?

Samenvatting van de ervaring: Ruimte om samen te vatten, te evalueren van de ervaring van de gezinszorg en socio-behandeling. Resumen de la experiencia: Espacio para resumir, evaluar la experiencia de la acogida familiar y trato socioeducativo.

Respecto a la documentación archivística localizada y catalogada, podemos decir que, además de la visita a otras bibliotecas y archivos de instituciones belgas, donde obtuvimos algún listado, noticias de prensa citadas en este artículo y referencias bibliográficas, fue una vez más el Amsab-Instituut voor Sociale Geschiedenis el principal yacimiento de información y de fuentes primarias: dibujos, fotografías, filatelia, correspondencia, noticias en prensa y carteles. Destaca la importante colección de 127 dibujos elaborados por los niños ${ }^{20}$ refugiados en el Grup Escolar Pau Claris de Barcelona, sufragado por la Generalitat de Catalunya, fruto de la donación de Emilia Labajos a este archivo de Gante. Estos excelentes e ilustrativos dibujos son una estupenda fuente iconográfica para comprender y complementar la explicación histórica, unos dibujos de media cuartilla realizados los días 13, 16, 27 y 28 de diciembre del 1938 y cuyos seis ejes temáticos propuestos por los educadores a los niños para que ilustraran la Guerra Civil vista por los ojos de la infancia son los siguientes: El bombardeig a l'escola; Privacions del menjar; La marxa del pare; Bombardeig; y Una alarma a l'escola.

20 GALLARDO CRUZ, José Antonio (2012) El dibujo infantil de la evacuación durante la Guerra Civil Española (1936- 1939), Universidad de Málaga: Servicio de Publicaciones; CABRA, Dolores (2004) «Aportaciones de A.G.E. a la memoria colectiva: los dibujos de los niños de la Guerra» en Ebre 38, No 2, p. 1-10; DUROUX, Rose (2006) «Ojos de niños sobre la guerra. La colección de dibujos de Françoise y Alfred Brauner» en Congreso La Guerra Civil Española 1936 - 1939. 


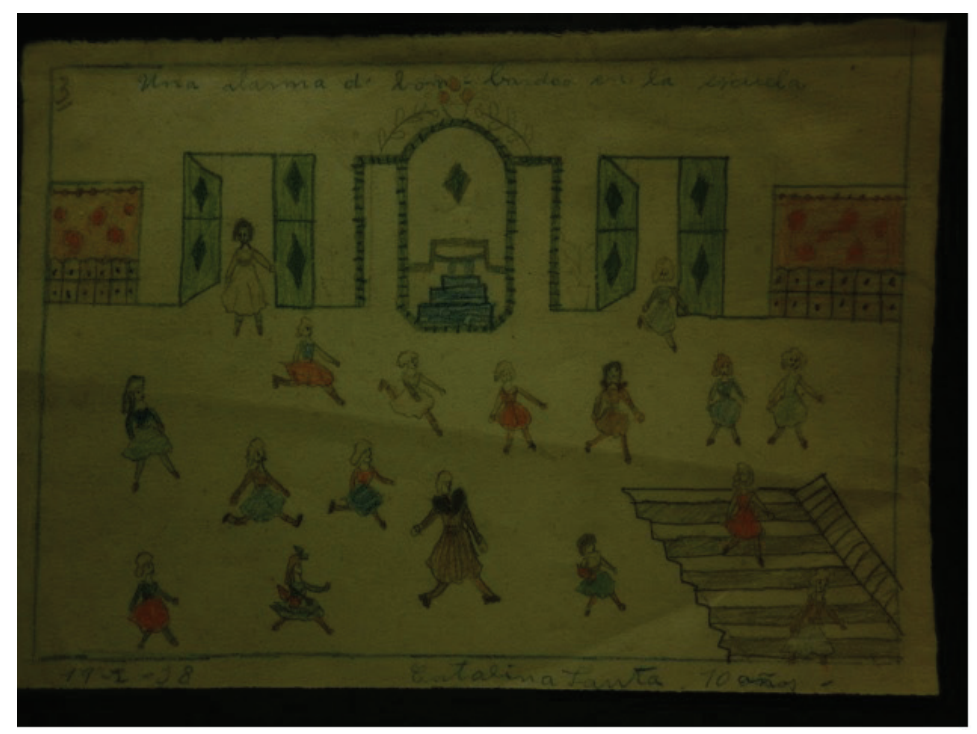

Alarma de bombardeo en la escuela, Amsab

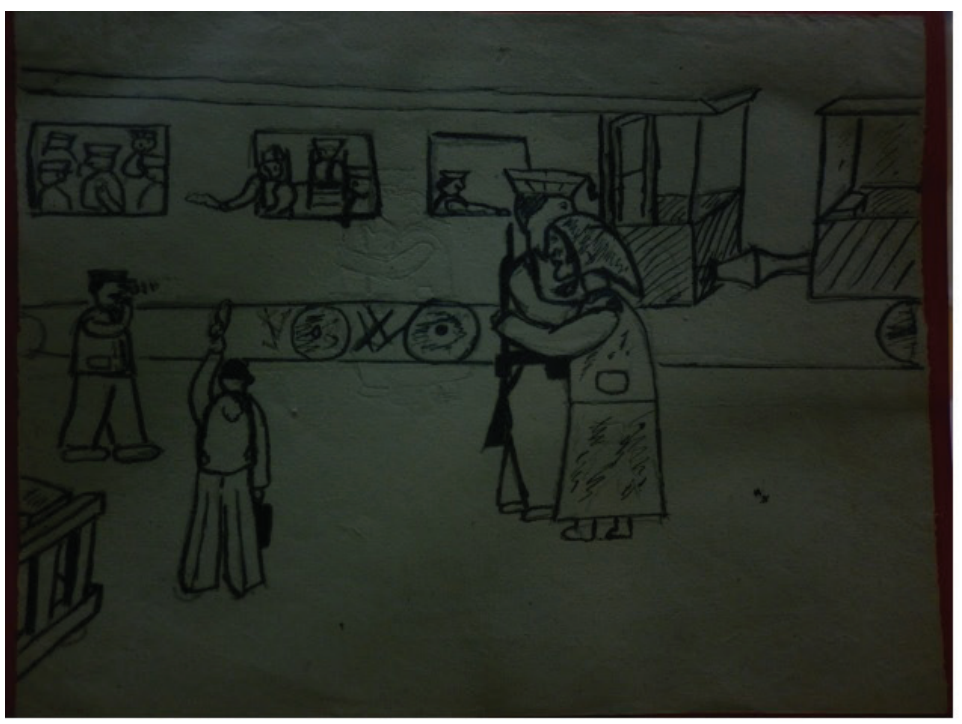

La despedida del padre al frente, Amsab.

De otro lado, el material más numeroso y variado es el correspondiente a las fotografías (dos cajas) de temática muy variada e interesante: manifestaciones contra la Guerra Civil, recepción de los niños exiliados en la estación de ferrocarril de Gante, visita de Margarita Nelken al Comité de Coordination d'aide a l'Espagne de Bruselas (16/12/1938), visita de Dolores Ibarruri (Pasionaria) al Stadspark Feest de Gante en septiembre de 1936, celebración del $1^{\circ}$ de mayo de 1938, niños con el puño en alto, grupo de niños en el Gravensteen (castillo) de Gante, festival de solidaridad con los niños españoles de junio de 1937, recepciones en Antwerpen, Moeskroen, Brugge, reconocimientos médicos a los 

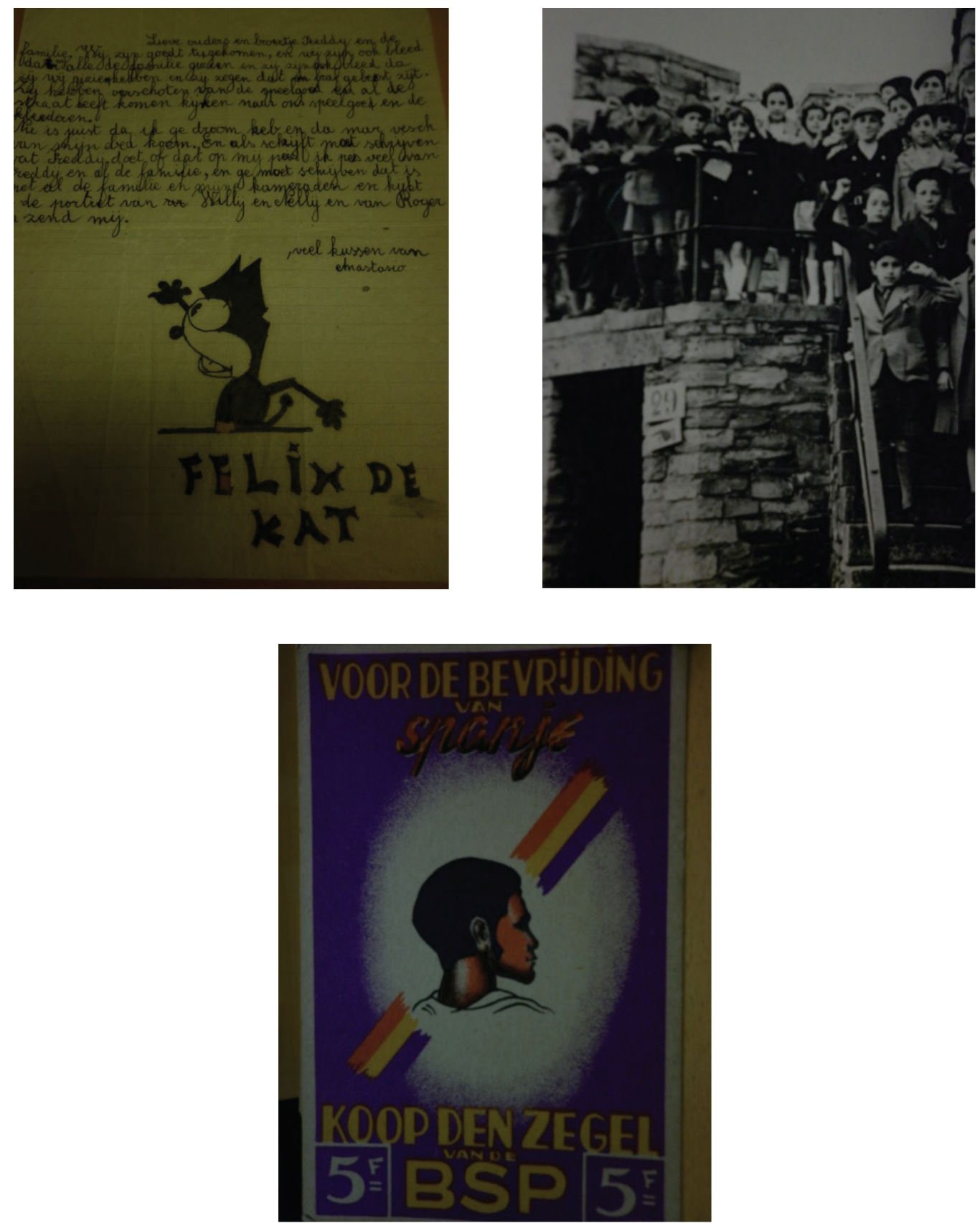

Muestra de la correspondencia, fotografías y sellos de los niños españoles en Bélgica, Amsab 
niños recién llegados de España, etc. Junto a estas fotografías que resultan una fuente histórica verdaderamente interesante para la temática, como ya demostrara Alicia Alted ${ }^{21}$, en el archivo del Amsab se encuentran depositadas también una colección de tarjetas postales que se comercialiaban para captar fondos destinados a la acogida de los nińos españoles en el exilio. En este caso, la variedad también es bastante grande: postales sobre la lucha de Barcelona (CNT/FAI), colección de postales «Les enfants d'Espagne au Home Emilie Vandervelde. Oostduinkerke» vendidas por 0'60 francos cada una «au profit des enfants d'Espagne», tarjetas del Ministerio de Propaganda, tarjetas postales editadas por el Departamento de propaganda de la delegación del Gobierno de Euskadi en Catalunya, tarjetas del Comissariat de propaganda de la Generalitat de Catalunya (Aeronáutica naval, Front d'Aragó i Des refugies dans le stade de Barcelone), tarjetas postales de las milicias antifascistas, postales del Comité Belge d'aide aux socialistes espagnols, tarjetas postales del Socorro Rojo Internacional, Postkaart National Komiteit het Spaansche volk (Brusel), etc. Toda una serie de tarjetas postales que junto a una colección de 10 sellos de correos Voor de Bevrijding van Spanje (Por la liberación de España) de 5 francos, constituyen un excelente material que se completa con la correspondencia personal de Anastasio Martin, un niño madrileño exiliado en Gante, que tras su retorno a España al finalizar la guerra civil, continua manteniendo el contacto con su familia adoptiva belga, o entre otros, los recuerdos de su primera comunión que envían Angelines y Luisito a sus padres adoptivos.

Como se puede fácilmente observar por la enumeración del material iconográfico, manuscrito y las historias de vida recogidas en esta investigación, la historia oral y material de nuestro pasado más reciente, puede ser una excelente fuente de iniciación a la investigación y de formación. La buena valoración de la experiencia por los alumnos que colaboraron en la recopilación de las historias de la infancia española exiliada en Gante, junto al material catalogado, demuestran el potencial pedagógico y de aprendizaje que éstos encierran para el mejor conocimiento de una historia común entre Bélgica y Espańa. En la actualidad nos encontramos estudiando y analizando con el rigor histórico y el tiempo que requiere, todo este material, del cual esperamos que pronto podamos realizar nuevas aportaciones que ayuden a recuperar parte de nuestra memoria histórica, pedagógica, social y de la infancia.

ALTED VIGIL, Alicia (2005) «El instante congelado del exilio de los niños de la guerra civil española» en DEP. Deportate, Esuli, Profughe, no 3, pp. 263-281. 